were defined by LV relative wall thickness and LV mass indexed to height (gram/height in $\mathrm{m} 2.7$ ). Multivariable logistic regression analyses were performed to define young adulthood determinants of LV geometric patterns.

Results The prevalence of normal geometry, concentric remodelling, eccentric and concentric hypertrophy were $79.0 \%, 7.6 \%, 8.7 \%$ and $4.7 \%$ respectively. Males showed significantly higher prevalence for concentric remodelling and eccentric hypertrophy than females (5.6\% vs $2.0 \%$ and $6.4 \%$ vs $2.3 \%, p<0.01$ ), however such differences were not noted for normal geometry and concentric hypertrophy ( $p>0.05)$. Using the normal geometry group as reference, individuals with eccentric and concentric hypertrophy showed significantly higher levels of BMI $\left(36.3 \mathrm{~kg} / \mathrm{m}^{2}\right.$ and $38.6 \mathrm{~kg} / \mathrm{m}^{2}$ vs $27.2 \mathrm{~kg} / \mathrm{m}^{2}$, $\mathrm{p}<0.001)$, SBP (127.5 mm Hg and $137.2 \mathrm{~mm} \mathrm{Hg}$ vs $114.7 \mathrm{~mm} \mathrm{Hg}$, $\mathrm{p}<0.001)$, DBP $(85.2 \mathrm{~mm} \mathrm{Hg}$ and $89.7 \mathrm{~mm} \mathrm{Hg}$ vs $73.5 \mathrm{~mm} \mathrm{Hg}$, $\mathrm{p}<0.01)$, glucose $(111.2 \mathrm{mg} / \mathrm{dl}$ and $129.3 \mathrm{mg} / \mathrm{dl}$ vs $85.2 \mathrm{mg} / \mathrm{dl}$, $\mathrm{p}<0.01)$, DM (24.3\% and $41.6 \%$ vs $4.3 \%, \mathrm{p}<0.001)$ and triglycerides $(156.8 \mathrm{mg} / \mathrm{dl}$ vs $128.5 \mathrm{mg} / \mathrm{dl}, \mathrm{p}<0.001)$ and total/HDL-C ratio (4.9 vs $4.1, p<0.01$ ) were higher significantly in eccentric hypertrophy only. However, none of these risk factors differed significantly between normal geometry and concentric remodelling groups ( $p>0.05)$. In Multivariable logistic regression models age, gender, BMI, SBP, DBP, glucose, DM, triglycerides and total/HDL-C ratio, male gender was related to concentric remodelling hypertrophy $(\mathrm{OR}=2.63,95 \% \mathrm{CI} 1.21$ to $5.64, \mathrm{p}=0.019)$, BMI was related to eccentric hypertrophy ( $O R=1.162,95 \%$ CI 1.08 to $1.20, p<0.001)$ and $\mathrm{DM}$ was related to concentric hypertrophy $(\mathrm{OR}=6.354,95 \% \mathrm{CI}$ 3.24 to $35.0, p=0.002$ )

Conclusions These findings showed that eccentric hypertrophy and concentric hypertrophy were more frequent and male gender, obesity and DM were significant determinants of these patterns of adverse cardiac remodelling in young adults.

\section{e0371 THE EFFECT OF ALDH2 GENETIC POLYMORPHISM ON MYOCARDIAL ISCHAEMIA REPERFUSION INJURY IN CHINESE}

doi:10.1136/hrt.2010.208967.371

Chen Hong, Zhang Fang-fang. Department of Cardiology Peking University People's Hospital

Background Recently, several animal studies investigated the relation between ALDH2 and cardiac ischaemia /reperfusion injury, but the results were controversial. Meanwhile, no relevant researches on population have been reported. And It is well known that acetaldehyde dehydrogenase 2 (ALDH2) has a significant variation in a single-nucleotide polymorphism of so-called G487A polymorphism in Asian, where the mutant allele is carried by nearly $50 \%$ of east Asians which has significant reduced or completely lost catalytic activity than people with ALDH $2 * 1 /{ }^{*} 1$ genotype.

Objective To investigate the association between ALDH2 G487A polymorphism and myocardial ischaemia/reperfusion injury in Chinese.

Methods We serially measured the release of troponin I (cTNI) and creatine kinase $\mathrm{MB}(\mathrm{CKMB})$ in 148 patients with acute myocardial infarction. The extent of cardiac injury was divided into two categories, the larger one of which is determined when the peak level of myocardial enzymes exceeded $30 \mathrm{ng} / \mathrm{ml}$ and $80 \mathrm{mg} / \mathrm{ml}$ for cTNI and $\mathrm{CKMB}$ respectively. Meanwhile, ALDH2 genotype was detected as well as other clinical parameters. Logistic regression analysis was used to analyse the association between the ALDH2 genotypes and myocardial ischaemia /reperfusion injury.

Results In 146 patients with acute myocardial infarction whose myocardial injury was estimated by cTNI $(\mathrm{p}=0.040)$ and in patients with STEMI undergoing PCI whose myocardial injury was estimated by cTNI $(n=72, p=0.018)$ and CKMB ( $n=67, p=0.035)$ respectively, the proportion of individuals with mutant allele was higher in patients with smaller injury than in that with larger. ALDH2 genetic mutation may be an independent protective factor for patients with acute myocardial infarction undergoing PCI (OR 0.264, $\mathrm{p}=0.034$ ) and patients with STEMI undergoing PCI (OR 0.264, $\mathrm{p}=0.034$ ) when injury was assessed by cTNI but not CKMB.

Conclusions ALDH2 G487A polymorphism is possibly associated with myocardial ischaemia/reperfusion injury in Chinese. ALDH2 geneic mutation (G487A) may confer independent cardioprotection in patients with acute myocardial infarction undergoing PCI and those with STEMI undergoing PCI.

\section{Q0372 POST-OPERATIVE OBSERVATION OF THE SAFETY AND ANGIOGENESIS EFFECT OF DIRECT CURRENT STIMULATION IN A MYOCARDIAL INFARCTION RABBIT MODEL}

doi:10.1136/hrt.2010.208967.372

Zhang Ping, Liu Zhitao, He Guoxiang, Liu Jianping, Feng Jian. Department of Cardiology, Southwest Hospital, The Third Military Medical University

Introduction The purpose of the current study was to evaluate the safety of low voltage direct current (DC) electric stimulation and its angiogenesis effect in a rabbit myocardial infarction (MI) model with electrodes directly fixed on the epicardium.

Materials and methods 28 Japanese white rabbits were randomly divided into control and treatment groups with 14 rabbits in each group. MI was induced by left anterior descending (LAD) artery ligation. A pair of platinum electrodes was directly placed on the ambilateral epicardium next to the LAD artery. Low voltage DC electric stimulation $(4.0 \mathrm{~V} / \mathrm{cm}, 60 \mathrm{~min} /$ day) was given to the treatment group immediately following the surgery until the 4th week post-operation. Parameters including blood routine, biochemistry, cardio and respiratory, pathology and immunohistochemisty from both groups were monitored throughout the experiment. Capillary density was counted at the end of the experiment.

Results The overall mortality rate was $7.1 \%$, pneumothorax rate was $3.6 \%$, and the intraoperative arrhythmia rate was $7.1 \%$. Transient hypotension, anaemia, leucocytosis, hypoxaemia and slight increase of the myocardium enzyme were observed in both control and treatment groups. Except minor inflammatory cell infiltration and mild hyperaemia, there was no other adverse response observed on the myocardium caused by electric thermal effect. The capillary density in the treatment group $(140.7 \pm 21.5)$ was significantly higher than that of the control group $(60.3 \pm 21.7)(p<0.001)$ at the end of the experiment.

Conclusion It is safe to apply low voltage DC electric stimulation to the MI rabbits in addition to promote the myocardium angiogenesis.

\section{e0373 INTRAVASCULAR ULTRASOUND STUDY ON ANGIOGRAPHIC CONTRAST MATERIEL DRAIN-LAGGED CORONARY SEGMENTS}

doi:10.1136/hrt.2010.208967.373

Cheng Xunming, He Guoxiang, Tong Shifei, Ran Boli, Liu Jianping. Dept. of Cardiology, Southwest Hospital, Third Military Medical University \& Chongqing Institute of Interventional Cardiology, Chongqing, China

Introduction To investigate the structural characteristics and its clinical significant of angiographic contrast materiel drain-lagged coronary segments. 